\title{
Influence of non-protein amino-acid mimosine in peptide conformational propensities from novel Amber force field parameters
}

\author{
Asier Urriolabeitia, ${ }^{\dagger}$ David De Sancho, ${ }^{\ddagger}$ and Xabier López ${ }^{*, \hbar}$ \\ $\dagger$ Department of Physical Chemistry, University of Zaragoza, Calle Pedro Cerbuna, 12, 50009, \\ Zaragoza, Spain \\ $\$$ Polimero eta Material Aurreratuak: Fisika, Kimika eta Teknologia, Kimika Fakultatea, \\ UPV/EHU \& Donostia International Physics Center (DIPC), PK 1072, 20080 Donostia-San \\ Sebastian, Spain \\ E-mail: xabier.lopez@ehu.eus
}

\begin{abstract}
Mimosine is a non-protein amino acid derived from plants known for its ability to bind to divalent or trivalent metal cations such as $\mathrm{Zn}^{2+}, \mathrm{Ni}^{2+}, \mathrm{Fe}^{2+}$ or $\mathrm{Al}^{3+}$. This results in interesting antimicrobial and anti-cancer properties, which make mimosine a promising candidate for therapeutic applications. One possibility is to incorporate mimosine into synthetic short peptide drugs. However, our understanding of how this amino acid affects peptide structure is still limited, reducing our ability to design effective therapeutic compounds. In this work, we used computer simulations to understand this question. We first build parameters for the mimosine residue to be used in combination with two classical force fields of the Amber family. Then, we used atomistic molecular dynamics simulations with the resulting parameter sets to evaluate the influence of mimosine in the structural propensities for this amino acid. We compared the results of these simulations with identical peptides where mimosine is replaced by either
\end{abstract}


phenylalanine or tyrosine. We found that the strong dipole in mimosine induces a preference for conformations where the amino acid rings are stacked over more traditional conformations. We validated our results using quantum mechanical calculations, which provide a robust foundation to the outcome of our classical simulations.

\section{Introduction}

Mimosine, or $\beta$-[N-(3-hydroxy-4-oxypyridyl)]- $\alpha$-aminopropionic acid, is a non-proteinogenic amino acid found in the members of Mimosoideae clade. ${ }^{1}$ It has been found to be a reversible inhibitor of DNA replication because of its ability to strongly bind to metals in the active site of many enzymes. ${ }^{2}$ Based on this activity, mimosine has been reported to have antimicrobial, antifungal ${ }^{3}$ and antiviral properties. ${ }^{4}$ Moreover, it has also found its way on therapeutic applications, showing anticancer activity ${ }^{5}$ and anti-inflammatory properties. ${ }^{6}$

One of us recently proposed mimosine containing peptides as decorporation agents of $\mathrm{Al}(\mathrm{III})$, a recognized neurotoxin. ${ }^{7}$ This proposal is based on the structural similarity of mimosine to deferiprone (DFP), a drug used for Fe(II) removal that has also shown promising results on treating the accumulation of high-valent metal cations, ${ }^{8}$ such as $\mathrm{Al}(\mathrm{III})$ and $\mathrm{Fe}(\mathrm{III})$. The proposed compounds had similar structures to that of deferoxamine (DFO), another drug used for Fe(II) decorporation (see Figure 1). DFO is able to bind to all six vacancies of the Fe(II) making it a very strong ligand. Studies have shown that DFO is a more effective decorporation agent than DFP. However, it suffers from other issues, such as being poorly absorbed and some secondary effects. ${ }^{9}$ Thus, the development of novel polypeptides able to strongly bind to all six vacancies of $\mathrm{Al}(\mathrm{III})$ can be a highly promising approach to creating decorporation agent candidates. ${ }^{10}$ However, the effects of this non-protein amino-acid in the context of a polypeptide chain have not been explored.

In this work we have studied the properties of mimosine and its influence on polypeptides containing it using atomistic molecular dynamics (MD) simulations. MD has become a standard tool for the characterization of structural propensities of peptides and disordered proteins in aqueous solution. ${ }^{11}$ However, for non-protein amino acids like mimosine, parameters for running simula- 


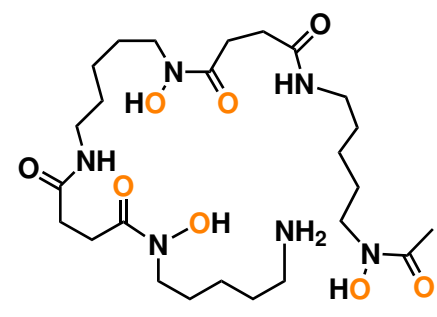

(A)

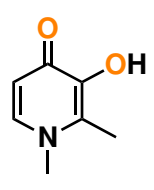

(B)

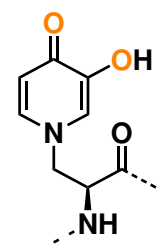

(C)

Figure 1: Structure of DFO (A), DFP (B) and the L-mismosine residue (C). Atoms in orange are the ones binding to metals.

tions are not widely available. To that end we have parametrized the mimosine residue for two widely used Amber force fields, ff99SB ${ }^{12}$ and ff03. ${ }^{13}$ We have then run simulations of two peptides of different lengths relevant as metal chelators, and studied the effects of mimosine residues. As reference, we also simulated peptides where the mimosine residue is replaced by one of its most similar protein amino acids, tyrosine and phenyl-alanine. For both force fields we found that mimosine exhibits in strong electrostatic interactions, that significantly influence the peptide behaviour. We validated our results against quantum mechanical calculations of these systems, which give a more detailed view of the energetics in mimosine peptides.

\section{Methods}

\section{Mimosine parametrization}

The parametrization of the mimosine amino acid was performed using the AmberTools14 package. ${ }^{14}$ Both the ff99SB and ff03 force fields include atom types that can be used to define the topology of every atom in the mimosine residue. Therefore, the original parameter sets of the force fields contain all necessary bonded and van der Waals parameters. Nonetheless, atomic charges for the residue had to be derived for both force fields. We followed the methods described by the force field developers as closely as possible. A minor change is that we replaced the geometry optimizations, originally done with prior force fields, by quantum mechanical optimizations (see Supporting Information). This small modification was first validated by deriving the charges of 
selected amino acids for both force fields. Specifically, we used our protocol for phenylalanine and tyrosine, which were chosen due to their structural similarity to mimosine, and asparagine and tryptophan, since both contain an $\mathrm{sp}^{2}$ nitrogen like that in the mimosine ring.

\section{Equilibrium molecular dynamics simulations}

To explore the effects of mimosine in the conformational preferences of short peptides we have run MD simulations of two model systems, with sequences Ace-XGPGXG-Nme and Ace-XGPGXGGXNme (see Figure 2). In these systems, $\mathrm{X}$ can be either the mimosine amino acid (Mms), Phe or Tyr, which we use for the purpose of comparison. Peptide chains were initially modelled as right handed $\alpha$-helices using the Molefacture plugin of VMD. ${ }^{15}$ They were placed in periodic orthorhombic boxes leaving $1 \mathrm{~nm}$ of margin in each direction. The systems were minimized using the steepest descent algorithm and then equilibrated in two stages. First, we run a 100 ps simulation in the NVT ensemble and then another $100 \mathrm{ps}$ in the NPT ensemble, both at $300 \mathrm{~K}$. Simulations were run using the velocity-rescaling thermostat and the Parrinello-Rahman pressure coupling ${ }^{16}$ to fix the pressure at 1 bar. Production MD simulations were run using the same conditions as the NPT equilibration. Dynamics were propagated for $500 \mathrm{~ns}$ and 3 replicas were run for each system using a 2 fs time step. Electrostatic interactions were calculated using PME $^{17}$ and the cutoffs for both electrostatic and van der Waals interactions were $1 \mathrm{~nm}$. All the MD simulations were performed using the ff99SB and ff03 force fields (including the mimosine amino acid) and the TIP3P water model ${ }^{18}$ using the Gromacs package (version 2018). ${ }^{19}$

\section{Metadynamics}

We ran metadynamics simulations using the PLUMED plugin for the version 2018.6 of GROMACS. ${ }^{20}$ Simulations were prepared and ran using the same methodology as the equilibrium dynamics. Metadynamics were initially ran for $30 \mathrm{~ns}$ and if the system had not converged, further extended for 5 additional ns, after which convergence was reassessed. A system was considered converged if both the height of the deposited gaussians and the free energy difference between the 
Ace-XGPGXG-Nme

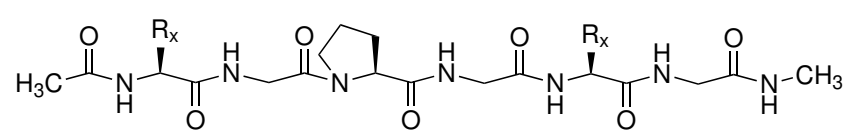

$$
\mathrm{X}=\mathrm{Mms}
$$

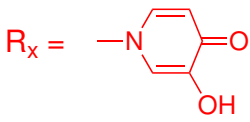

$\mathrm{X}=\mathrm{F} / \mathrm{Phe}$

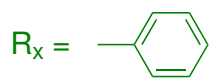

Ace-XGPGXGGX-Nme

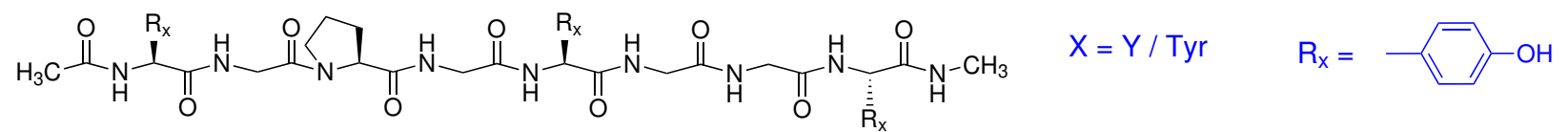

Figure 2: Peptides used in our equilibrium simulations to study the conformational preferences

two most stable conformations ( $\alpha_{\mathrm{R}}$ and $\beta$ ) showed no significant changes in the last $5 \mathrm{~ns}$ of the simulation. The collective variables studied were the backbone dihedral angles $(\Phi$ and $\Psi)$. The bias factor chosen was 6, gaussians were deposited every 500 steps ( 1 ps) and their width was 0.35 $\mathrm{rad}$ in each $\mathrm{CV}$ and started at a height of $1.2 \mathrm{~kJ} / \mathrm{mol}$. Matplotlib $3.1 .1^{21}$ and the metadynminer package of $\mathrm{R}^{\mathrm{a}}$ were used for plotting the results.

\section{DFT calculations}

All structures were optimized and characterized using Gaussian16 software ${ }^{22}$ employing the wB97XD density functional ${ }^{23}$ in conjunction with the $6-311++\mathrm{G}(\mathrm{d}, \mathrm{p})$ basis set for all atoms ${ }^{24-31}$ and taking into account aqueous solvation effects using the Polarizable Continuum Model (PCM) approach ${ }^{32}$ The characterization of fully optimized structures confirmed all minima have no imaginary frequencies.

Electronic and solvation energies were further refined by single-point calculations at the wB97XD/6$311++\mathrm{G}(3 \mathrm{df}, 2 \mathrm{p}) / \mathrm{PCM}$ (water) level of theory. hereinafter referred to as DFT level of theory. Taking into account these energies, we evaluated the interaction energies $\Delta E_{\text {int }}$ between the rings that form the sidechains of Mms, Tyr and Phe as the difference in energy between the ring dimers and the

\footnotetext{
ahttps://www.rdocumentation.org/packages/metadynminer
} 
infinitely separated rings. For instance

$$
\Delta E_{\text {int }}=E_{\mathrm{X}-\mathrm{X}}-2 \times E_{\mathrm{X}} \quad ; \quad \mathrm{X}=\mathrm{Mms}, \text { Phe, Tyr }
$$

We also select some snapshots from the MD simulations and calculated the interaction between the stacked ring dimers by constrained optimizations, in which the rings were forced to keep the same distance and relative orientation as in the MD structure. This was done by freezing the relative distance between the nitrogen atoms of the mimosine rings and fixing the dihedral formed between OC-N2-N2-CO atoms. For Phe rings analogous constrains were introduced. Finally, an assessment of the stabilization of these structures introduced by the presence of Mms rings is made by full quantum calculations of a simplified model of the model pentapeptide.

\section{Results and discussion}

In this section we present and analyze the results obtained in this work. Firstly, we assess the validity of the charge derivation methods and examine the charges obtained for the mimosine. Secondly, we present the results obtained from metadynamics calculations to study the torsional propensities of this amino acid. Then we introduce the data from simulations of Mms-containing polypeptides and compare them to simulations of analogues obtained by substituting the Mms by Phe or Tyr. Finally, we validate our results against quantum mechanical calculations.

\section{Mimosine charge derivation}

The charge derivation methods used for the Mms replicated the methods used for the protein amino acids while substituting the processes that required data from prior force fields by QM calculations. We evaluated their results by comparing the charge distributions obtained for four representative residues against those published by the authors of the force fields. As shown in Figure 3, we obtain excellent agreement between the existing parameters and those we have obtained with the 
slightly modified version of the protocol for charge derivation. These results confirm the adequacy of the methodology applied in this work to produce a set atomic charges consistent with the Amber methodology.

We then moved on to produce charges for the Mms residue, which we compare in Figure 4 with those for Phe and Tyr. For both force fields, we observe similar trends in the charge distributions. The aromatic rings of Phe and Tyr show evenly distributed negative charges among the carbons and positive among the hydrogens bonded to them. Whereas carbons bonded to the $\beta$ carbon or electron withdrawing groups show more positive charges. This effect is also observed for Mms, but to a greater extent, with the carbons bonded to the hydroxy and oxo groups positively charged, especially the latter one. Furthermore, the N2 nitrogen atom of the Mms ring shows a more positive charge than those of its analogue carbons in Phe and Tyr, which can be correlated to the nitrogen donating two $p$-electrons by resonance, rather than the one donated by the carbons. These positive charges are balanced by the neighboring atoms, leading to significantly more negative charges in the $\mathrm{CD}$ and $\mathrm{CE}$ atoms. While these distinctions for the Mms charges can be observed for both force fields it should be noted that they are heightened for ff99SB.
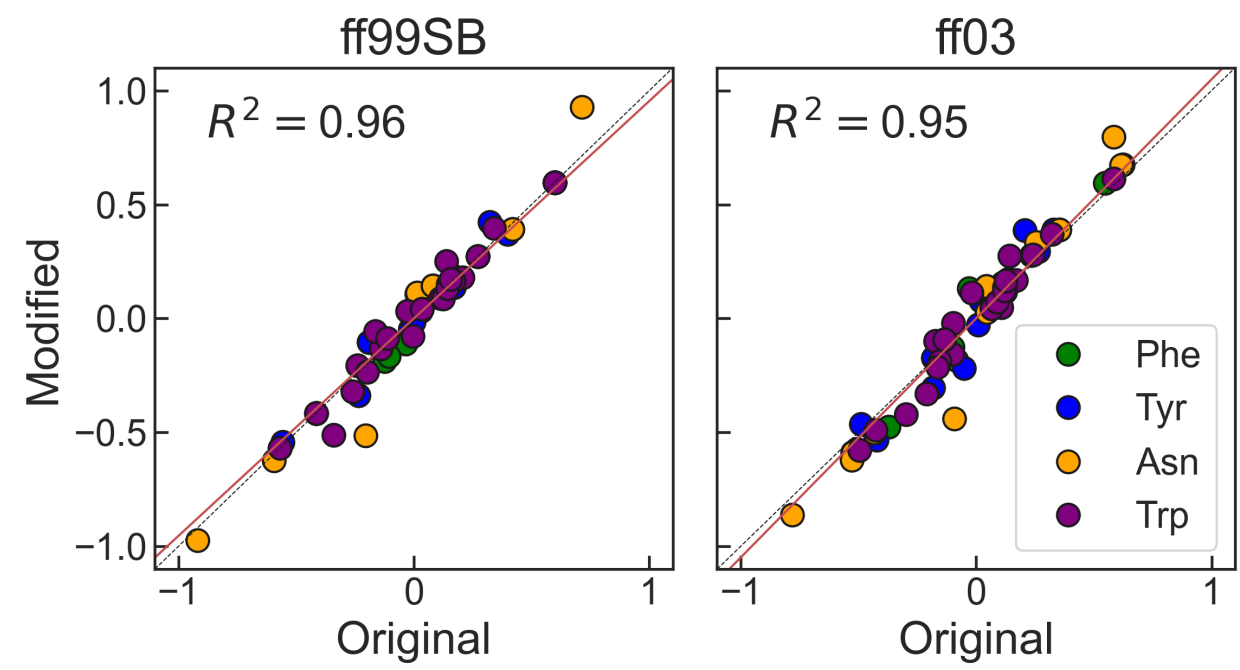

Figure 3: Correlation between original and derived charges with the modified workflow for the Amber ff99SB (left) and ff03 (right) force fields. We show results for Phe, Tyr, Asp and Trp. The dashed black line marks the identity line and the red line is a linear fit to the data. 
ff99SB
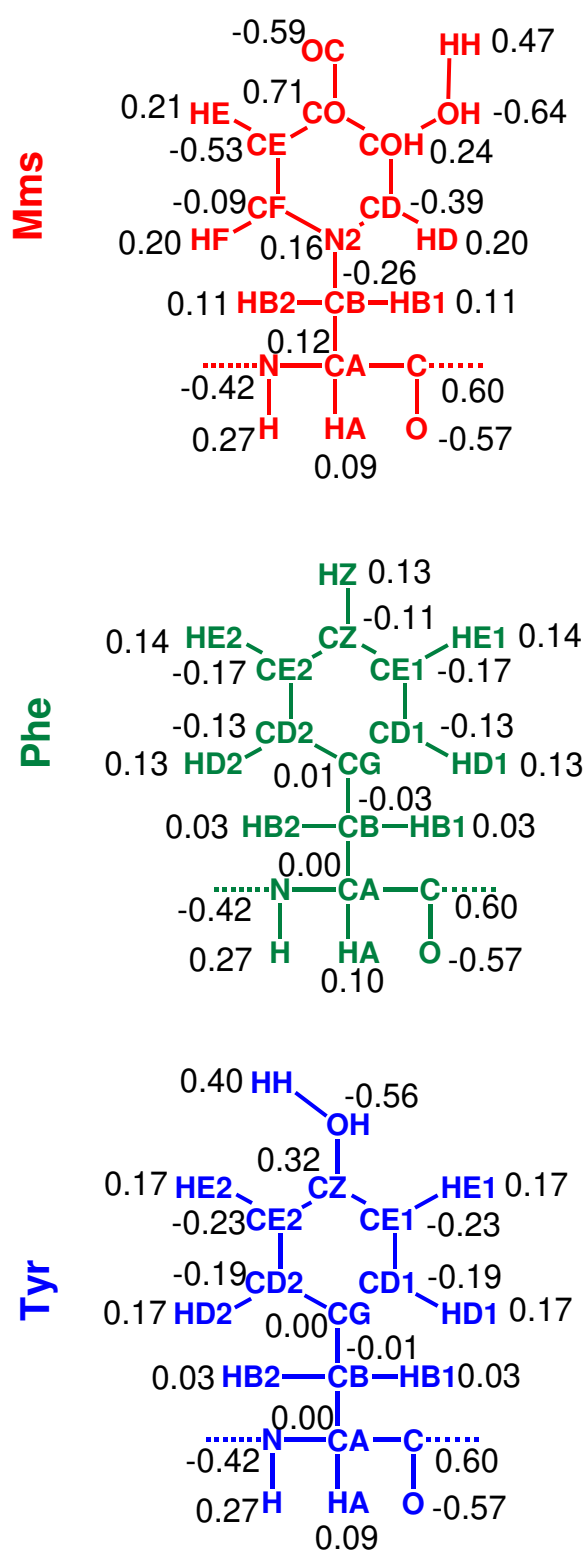

ff03
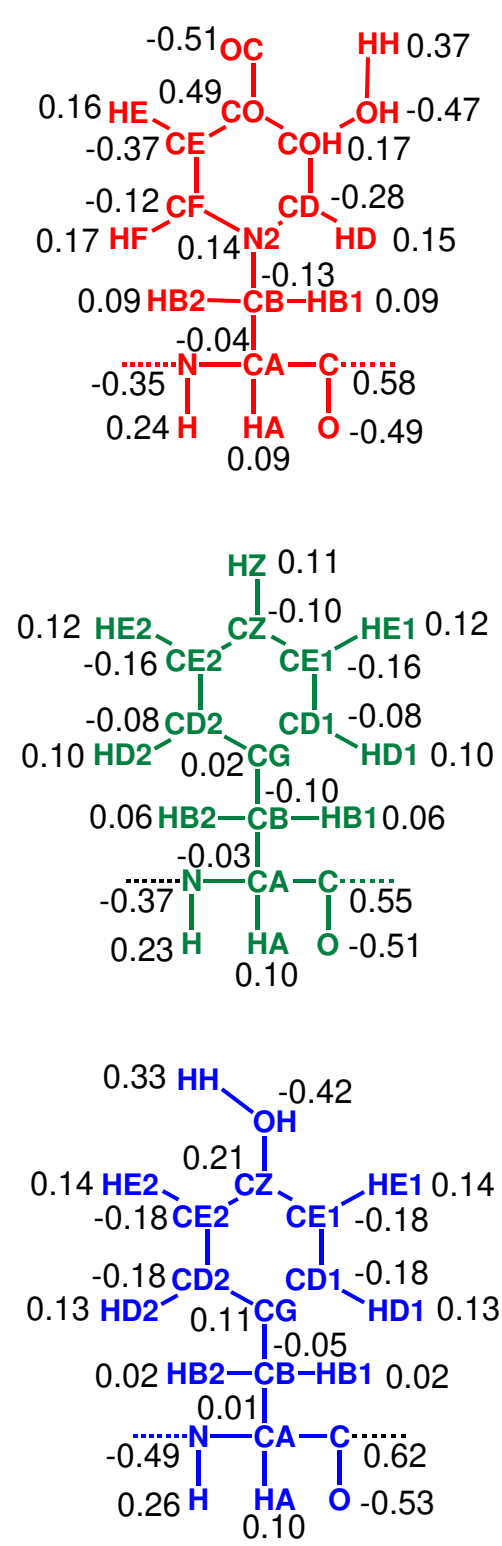

Figure 4: Calculated partial charges for the Amber ff99sb and ff03 force fields for Mms, Phe and Tyr.

\section{Free energy landscape of the mimosine dipeptide}

In order to understand the differences between the mimosine residue and the most similar protein amino acids, we used the new set of parameters in molecular simulations. Specifically, we performed metadynamics simulations on the terminally blocked peptides (often termed dipeptides) of Mms, Phe and Tyr to estimate their free energy surfaces as a function of the backbone dihedral 
angles, $\Phi$ and $\Psi$ (see Figure 5). For the three dipeptides and for both the ff99SB and ff03 force fields we find very similar free energy surfaces suggesting only subtle differences in the backbone conformational preferences. In all cases, the PPII $/ \beta$ conformation is the most stable one, except for the tyrosine dipeptide using ff03, where the $\alpha_{\mathrm{R}}$ minimum is $0.7 \mathrm{~kJ} / \mathrm{mol}$ lower in free energy (see Table 1). As expected, the $\alpha_{\mathrm{L}}$ conformation is significantly less stable than the $\beta$ and $\alpha_{\mathrm{R}}$ ones, with notably lower energetic differences in the case of mimosine. Additionally, for both force fields we find that the free energy barrier between the PPII/ $\beta$ and $\alpha_{\mathrm{L}}$ wells is notably lower than for both Phe and Tyr (see Table 2), which should result in more frequent transitions to sample this free energy basin for this non-protein amino acid .

Table 1: Relative free energy in $\mathrm{kJ} / \mathrm{mol}$ of the $\alpha_{\mathrm{R}}$ and $\alpha_{\mathrm{L}}$ conformations relative to the PPII/ $\beta$ for the Phe, Tyr and Mms dipeptides for the ff99SB and ff03 force fields.

\begin{tabular}{|cccc|}
\hline & Mms & Phe & Tyr \\
\hline \hline \multicolumn{4}{|c|}{ ff99SB } \\
\hline$\alpha_{\mathrm{R}}$ & 3.5 & 5.1 & 3.4 \\
$\alpha_{\mathrm{L}}$ & 8.2 & 12.4 & 11.5 \\
\hline \multicolumn{4}{|c|}{ ff03 } \\
\hline$\alpha_{\mathrm{R}}$ & 2.5 & 1.2 & -0.7 \\
$\alpha_{\mathrm{L}}$ & 11.9 & 21.1 & 20.6 \\
\hline
\end{tabular}

Table 2: Free energy barriers in $\mathrm{kJ} / \mathrm{mol}$ from the most stable conformation for the Phe, Tyr and Mms dipeptides for the ff99SB and ff03 force fields.

\begin{tabular}{|lccc|}
\hline & Mms & Phe & Tyr \\
\hline \hline \multicolumn{4}{c}{ ff99SB } \\
\hline $\mathrm{PPII} / \beta \rightarrow \alpha_{\mathrm{R}}$ & 10.5 & 13.9 & 13.4 \\
$\mathrm{PPII} / \beta \rightarrow \alpha_{\mathrm{L}}$ & 26.4 & 36.8 & 36.1 \\
\hline \multicolumn{5}{c}{$\mathrm{ff03}$} \\
\hline $\mathrm{PPII} / \beta \rightarrow \alpha_{\mathrm{R}}$ & 11.4 & 15.4 & 14.8 \\
$\mathrm{PPII} / \beta \rightarrow \alpha_{\mathrm{L}}$ & 20.1 & 34.8 & 34.5 \\
\hline
\end{tabular}

\section{Conformational preferences of mimosine in short peptides}

Having estimated the free energy landscapes for the capped amino acids, we now evaluate the behaviour of Mms in relation to Tyr and Phe in two short peptides with enhanced antimicrobial 


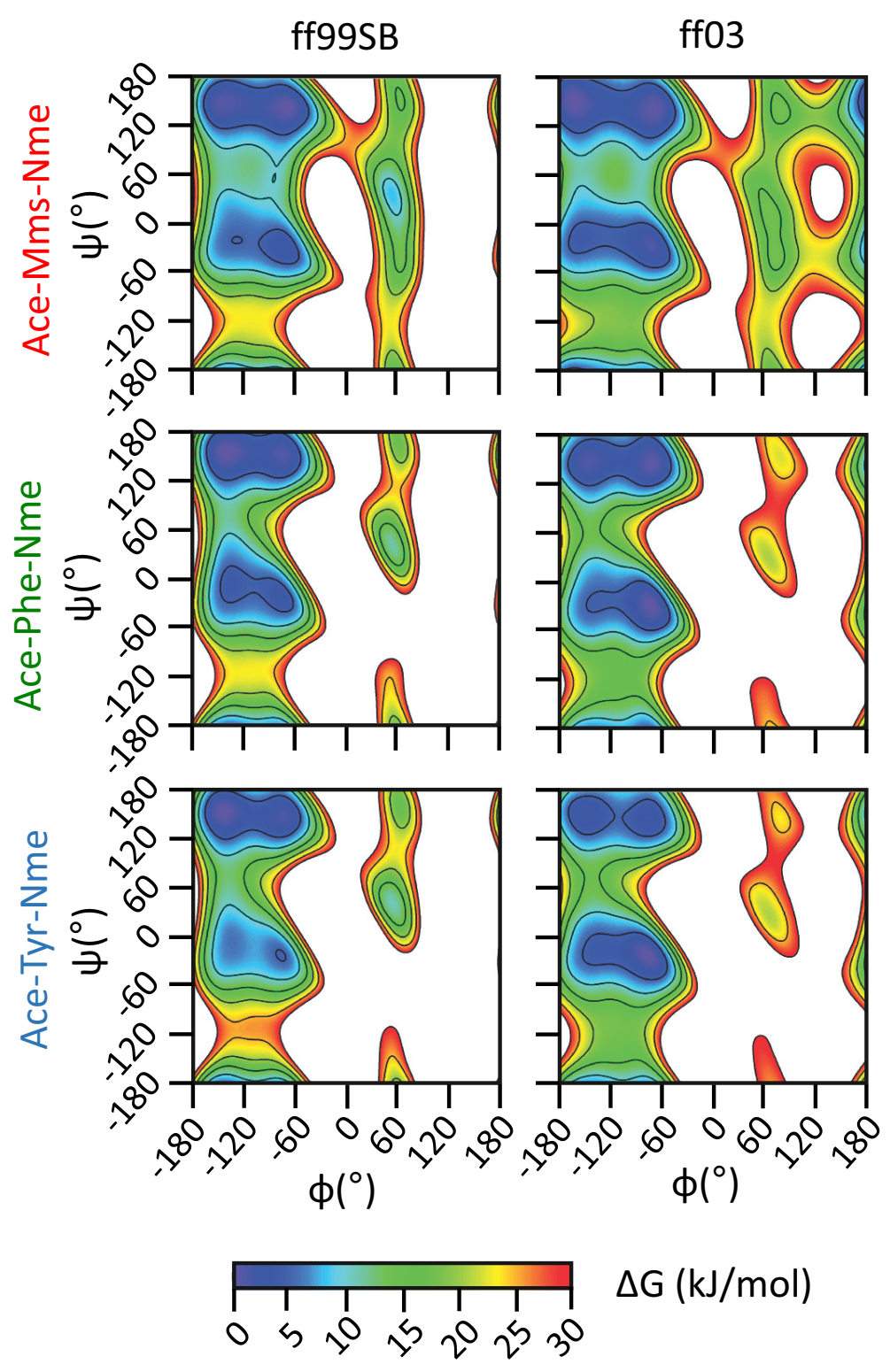

Figure 5: Ramachandran free energy landscapes for Phe, Tyr and Mms dipeptides calculated using the ff99sb and ff03 Amber force fields.

activity ${ }^{10}$ for both ff99SB and ff03 (see Figure 2). The first set of molecular dynamics corresponds to an hexapeptide with sequence Ace-XGPGXG-Nme, where X can be either mimosine, pheny- 
lalanine or tyrosine (see Methods). To characterize the dimensions of these peptides we estimate the distribution of the radius of gyration $\left(R_{g}\right.$, see Figure 6). We find that the distributions differ significantly between the Mms hexapeptide and its analogues including Phe and Tyr. Specifically, the distributions of $R_{g}$ values of the mimosine peptide are narrower and are centered at higher radius values than those of the Phe/Tyr peptides, suggesting the prevalence of conformations with a more expanded chain for the Mms peptide. The maxima for both force fields in the case of the mimosine hexapeptide are at $\sim 0.51 \mathrm{~nm}$ and the relative frequencies reached are considerably higher than those observed for either the Phe or Tyr peptides, with over $58 \%$ and $41 \%$ of the population being between 0.49 and $0.53 \mathrm{~nm}$ for ff99SB and ff03, respectively. While the distribution is slightly broader for ff03, the same trend can be observed for both force fields.

This result suggests that geometries that are not particularly important for Phe/Tyr peptides, become much more favourable for the Mms peptide. Further inspection of the trajectories revealed that mimosine significantly favours conformations where the peptide rings can interact. This is captured by the maxima around $0.44 \mathrm{~nm}$ in the distribution of inter-ring distances, shown in Figure 6. The same trends can be observed for both force fields, although to a different degree, with the preference for short inter-ring distances being more prominent for ff99sb. Additionally, a second maximum in the population appears at distances around $0.84 \mathrm{~nm}$, which dominates in the Phe/Tyr peptides, and is stabilized mainly by backbone interactions.

Therefore, the hexapeptide presents two dominant conformations, one stabilized by interactions between the Mms rings and a second one stabilized by interactions in the backbone leaving its Mms rings at longer distances. On the other hand, the Phe and Tyr hexapeptides show only one prevalent conformation, the latter one. To understand the interactions between the Mms rings, we analysed their relative orientations. In Figure 6 we show the distribution of the angle $\theta$ between the rings, which is drastically different in the mimosine peptide from that in both the Phe and Tyr peptides.

All of the results can be related to the strong interactions found in the out-of-register stacking of $\mathrm{Mms}$ rings. The positively charged $\mathrm{COH}$ atom can interact with the negatively charged $\mathrm{CD}$ and $\mathrm{CF}$ atoms. Similarly, the interactions of the $\mathrm{CO}$ and $\mathrm{OC}$ atoms in the different rings also favor this 

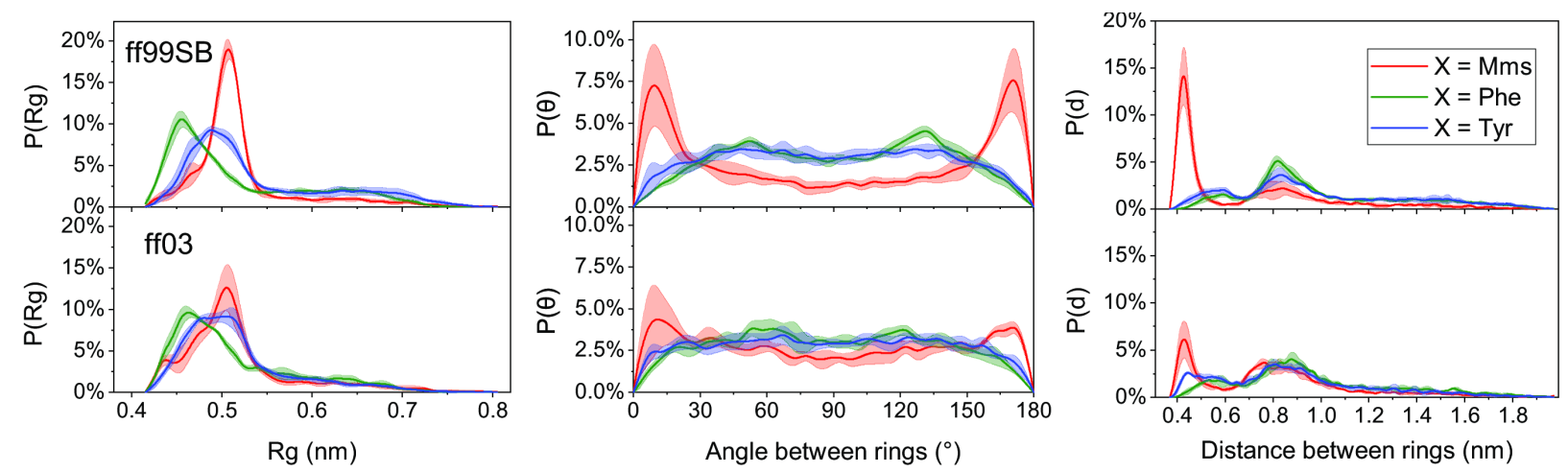

Figure 6: MD results for the hexapeptide. Left: Distribution of radii of gyration values. Center: Distributions of average distances between every heavy atom rings for Mms, Phe and Tyr. Right: Distributions of average angle between planes defined by the rings of Mms, Phe and Tyr. In all cases, we show results for X being Mms, Phe and Tyr and for both the ff99SB and ff03 force fields.

stacking pattern. On the contrary, the stacking of Phe and Tyr rings is considerably less favored. Therefore, the stacking of rings will be a significant driving factor of the conformations adopted by the mimosine hexapeptide while for Phe and Tyr it has little or no influence. The most stabilizing interactions for these peptides are hydrogen-bonds between the amide groups in the backbones. These interactions give raise to $\beta$-strand-like conformations in which the rings are separated corresponding to the maxima centered at $0.84 \mathrm{~nm}$. The greater freedom of movement of the rings in these conformations explains why these maxima are considerably broader than those corresponding to conformations with their rings tightly stacked. These $\beta$-strand-like conformations will also be observed for the mimosine hexapeptide, as the interactions between backbone groups compete with the stabilization of the MMS ring stacking. The differences between the results observed for the mimosine peptide in the two used force fields could be correlated with the MMS ring charges derived for ff03 being considerably lower than those obtained for ff99SB. Thus, the electrostatic interactions that favor the ring stacking are weaker for this force field.

We have run an additional set of simulations on a slightly longer peptide with sequence AceXGPGXGGX-Nme (see Fig. 2). In this case, the focus was on the stacking effect between their rings. In Figure 7 we show the distribution of average distances between the heavy atoms in the rings of the studied peptides for the ff99SB and ff03 force fields, respectively. For convenience, we label the rings 1 to 3 going from the $\mathrm{N}$ to the $\mathrm{C}$-terminus. As observed for the hexapeptide, 

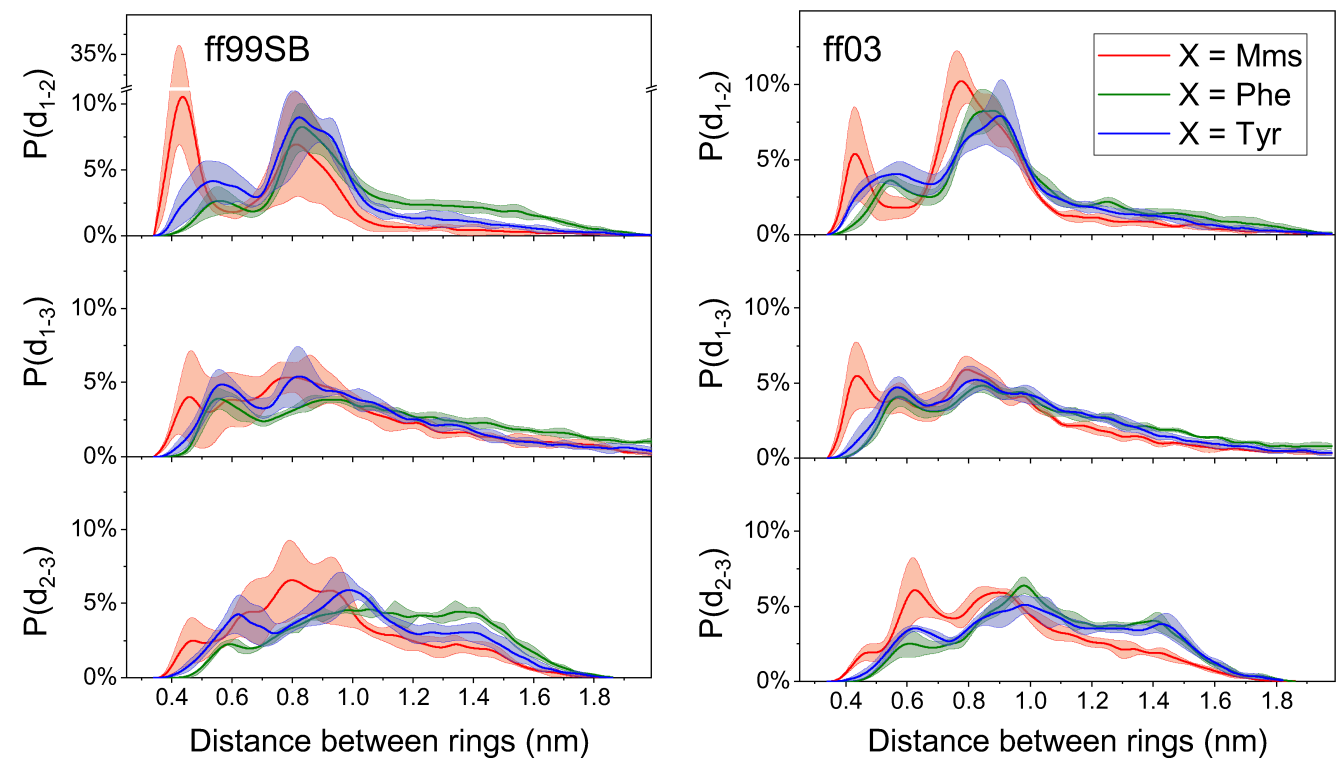

Figure 7: Distribution of inter-ring distances for rings 1 and 2 (top), 1 and 3 (center) and 2 and 3 (bottom) radii of gyration values, for octapeptides using the ff99SB (left) and ff03 (right) force fields.

the Mms-containing octapeptide behaves in a significantly different manner than its analogues favouring conformations in which Mms rings are stacked. As observed for the hexapeptide, the Mms ring stacking is less prevalent when using ff03. The unique behaviour triggered by the Mms residues is also present in the results of the other two ring pairs.

\section{Quantum Chemical Calculations on Mimosine Dimers}

To provide insight into the interaction energy between the mimosine rings, we performed Density Functional Theory (DFT) calculations (see Methods). First, we analyze the interaction energies for the sidechain models of Phe, Tyr, and Mms (See Figure 8). We started the optimization from two different ring orientations, in which the rings lie parallel or antiparallel to each other; namely, the methyl substituents, representing the beta carbon, lie at the same or opposite sides. Departing from the parallel orientation, we found significant differences among the three dimers. In the case of Phe-Phe dimer, we found both parallel and antiparallel structures with small differences in $\Delta E_{\text {int }}-5.5 \mathrm{kcal} / \mathrm{mol}$ versus $-5.1 \mathrm{kcal} / \mathrm{mol}$, respectively. However, in the case of Tyr, the stacking 
between the rings in the parallel conformation is disturbed to form a hydrogen bond, with a $\Delta E_{\text {int }}$ of $-6.3 \mathrm{kcal} / \mathrm{mol}$, whereas the antiparallel conformation maintains the ring stacking although the interaction is slightly weaker $-5.7 \mathrm{kcal} / \mathrm{mol}$. In the case of mimosine, the optimization that started from the parallel conformation led to the relative rotation of the rings yielding the antiparallel structure, with a significant interaction energy of $-9.1 \mathrm{kcal} / \mathrm{mol}$. Therefore, the stability order of the dimers is Mms-Mms > Tyr-Tyr $>$ Phe-Phe. These differences in energy are even greater when gas-phase calculations are considered with $\Delta E_{\text {int }}$ Mms-Mms (-17.0 kcal/mo) $>$ Tyr-Tyr (-8.4 $\mathrm{kcal} / \mathrm{mol})>$ Phe-Phe $(-6.2 \mathrm{kcal} / \mathrm{mol})$.

For Tyr and Mms dimers, we also considered the effect of adding explicit waters in the calculations. In this case, we evaluate the interaction energy of the dimers with respect to the monomer composed of one ring hydrogen-bonded to a water molecule. Both Tyr-Tyr and Mms-Mms systems can interact through hydrogen bonds with these waters. Still, the interaction of mimosine rings is more efficient since it can act both as a hydrogen donor and acceptor through the alcohol and carbonyl oxygens at the same time. Interestingly, the interactions with water molecules stabilize the parallel orientation of the mimosine rings, obtaining the overall stronger interaction among dimers, $-14.1 \mathrm{kcal} / \mathrm{mol}$, since both water molecules can adopt a geometry in which they also interact through a hydrogen bond. Notice, however, that even in the case of the antiparallel Mms-Mms structure, the addition of water molecules also has a stabilizing effect, with interaction energy of $-13.4 \mathrm{kcal} / \mathrm{mol}$. The $\Delta E_{\text {int }}$ in both Mms-Mms dimers is significantly higher than in Tyr-Tyr dimers.

In summary, Mms dimers show larger interaction energies than Phe or Tyr dimers. We also observed that the dipole of each mimosine ring is $9.7 \mathrm{D}$, which would explain the strong interaction energy in these stacked structures and the reorientation from parallel to antiparallel conformation in the optimization procedure, in the absence of explicit water molecules. Therefore, there is a inherent preference for an antiparallel orientation of both Mms rings. However, the presence of explicit water molecules and the resultant hydrogen bonding network can lead to a substantial stabilization of the parallel orientation. 


Phe--Phe

Figure 8: $\Delta E_{\text {int }}$ Interaction energies from DFT calculations for the interactions of the ring dimers in solution for parallel and antiparallel orientations. Values in parenthesis correspond to gas phase calculations. For Tyr-Tyr and Mms-Mms dimers we also consider the effect of adding explicit water molecules. 


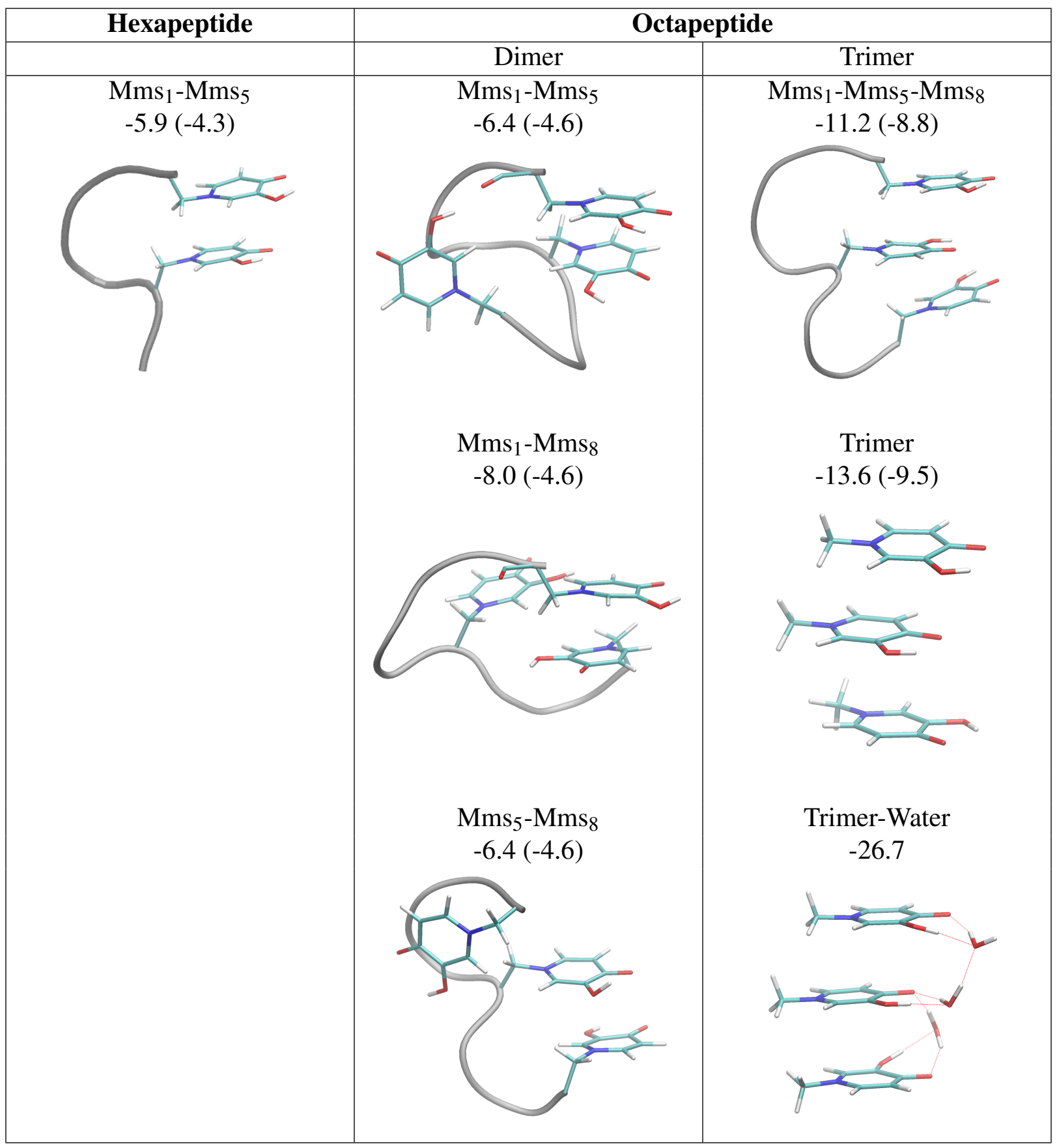

Figure 9: $\Delta E_{\text {int }}$ Interaction energies in solution at DFT level of theory for the Mimosine (Mms) dimers and trimers extracted from the hexa and octapeptide selected structures, and for analogous phenyl ring (Phe) dimers and trimers in parenthesis. Energies in kcal/mol. For the trimers, we considered constrained optimizations and unconstrained ones (trimer and trimer-water structures). 


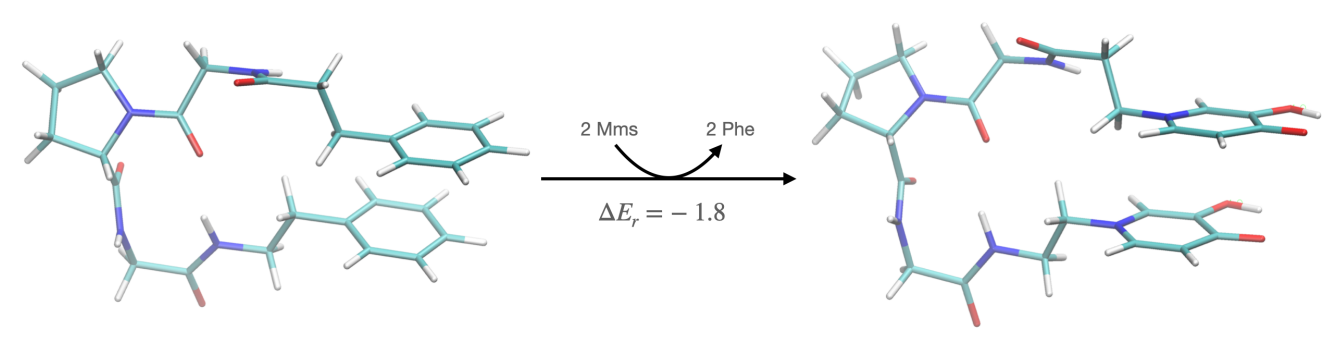

Figure 10: Substitution reaction energy, in $\mathrm{kcal} / \mathrm{mol}$, showing the stabilisation introduced when mimosine rings substitute phenyl rings in these structures. We have considered a reduced model of the hexapeptide which maintains only the backbone between the residues containing both rings. Energies calculated at the DFT level of theory.

\section{Relevance of Ring Stacking from QM Calculations}

The interaction between mimosine rings inside the peptide structure could be frustrated due to the constraints imposed by the peptide backbone. Therefore, one may wonder whether the inherent strong stacked interactions between mimosines are also significant in the peptides. To prove that, we have selected some snapshots from the MD dynamics for the mimosine hexa and octapeptides. We have performed optimizations of the ring dimers constrained to maintain the distance and relative orientations between the rings found in the peptides. Results are summarised in Figure 9. For comparison, we also evaluated the interaction energy of Phe-Phe dimers with analogous constraints. In all cases, the interaction between two mimosine rings was significantly higher than the interaction between two phenyl rings by roughly $1.5 \mathrm{kcal} / \mathrm{mol}$ for the interaction between $\mathrm{Mms}_{1}-\mathrm{Mms}_{5}$ and $\mathrm{Mms}_{5}-\mathrm{Mms}_{8}$ dimers. In the case of the $\mathrm{Mms}_{1}-\mathrm{Mms}_{8}$ interaction, we also found a structure with mimosine sidechains in an antiparallel conformation, which led to the highest interaction among the dimers $-8.0 \mathrm{kcal} / \mathrm{mol}$, almost double the interaction between phenyl dimer analogs, $-4.6 \mathrm{kcal} / \mathrm{mol}$. In addition, we considered DFT calculations with a reduced model of the mimosine hexapeptide (see Figure 10), which maintains the backbone atoms connecting both rings. Then, a substitution reaction energy is calculated for the exchange between mimosine and phenyl sidechains. The resultant reaction energy $\Delta E_{r}=-1.8 \mathrm{kcal} / \mathrm{mol}$ reveals a significant stabilization by mimosine dimer interaction. Notice that the stabilization is very similar to the one obtained by the constrained minimizations, namely $\Delta \Delta E_{\text {int }}=-1.6 \mathrm{kcal} / \mathrm{mol}$. However, these estimations of 
the stabilization of stacking conformations of mimosine rings should be considered a lower limit, since as we have seen before, stacked mimosine rings can also be further stabilized by a favorable hydrogen-bond network with water molecules, a feature not possible for phenyl rings.

We also observed the formation of mimosine stacked trimers with an interaction energy of $-11.2 \mathrm{kcal} / \mathrm{mol}$. If the constraints in geometry optimization are removed, we obtain an interaction energy of $-13.6 \mathrm{kcal} / \mathrm{mol}$, maintaining a similar structure and ring orientation. The interaction of these mimosine trimers is $4 \mathrm{kcal} / \mathrm{mol}$ stronger than analogous phenyl trimers. Finally, when considering explicitly the presence of water molecules, we observed a significant synergy in stabilization between stacked configuration and hydrogen bonded network, with an interaction energy of $-26.7 \mathrm{kcal} / \mathrm{mol}$ with respect to three separated mimosines, each one hydrogen-bonded to a water molecule.

In summary, although the peptides introduce constraints to the spatial arrangements that mimosine sidechains can adopt, stacked mimosine configurations like those observed in our classical simulations with the new Amber force field parameters are still prevalent and introduce significant stabilization. These stacked interactions are stronger than the ones for analogous Phe sidechains. Our DFT calculations suggest that they are stabilized by the strong interaction between large dipole-containing mimosine rings and a stable hydrogen bond network with water molecules.

\section{Conclusions}

In this study, we have produced force field parameters for the non-protein amino acid mimosine, with specific emphasis in the generation of new sets of charges for both Amber force fields ff99SB and ff03 using validated methods. We have first run metadynamics simulations on terminally blocked amino acids to estimate the Ramachandran free energy surfaces using the new parameters, and compared them with similar calculations for Phe and Tyr. When performed on the Mms dipeptide these simulations revealed a similar free energy landscape to that of the Phe and Tyr for both ff99SB and ff03. While all three dipeptides had the $\beta$ and $\alpha$ as their most stable conformations, 
Mms showed remarkably lower barriers, which may lead to faster conformational transitions. Although Mms showed a relatively more stable $\alpha_{\mathrm{L}}$ conformation it was not stable enough to play a role in the behavior of the peptide unless an external effect significantly stabilizes it.

Then, we assessed the effect of mimosine in the conformational preferences of short peptides, relative to its most similar amino acids, Phe and Tyr. In contrast to both of them, we observed the off-register stacking of the Mms rings, due to the higher atomic charges on its ring and $\beta$ carbon. The MD simulations showed that polypeptides containing various Mms amino acids in their sequence go under considerably less conformational changes than their analogues. This is caused by the highly favored Mms ring stacking. Most conformations that these polypeptides adopted throughout the simulations had their rings stacked, severely limiting the diversity of observed conformations. In the ff99SB force field the ring stacking of Mms peptides separated by the Gly-Pro-Gly chain the ring is favoured over others, dictating the behavior of the polypeptides containing this sequence. In the ff03 force field multiple ring stacking arrangements are prevalent, resulting in a greater variability of conformations.

Quantum chemical calculations at the Density Functional Theory confirmed the favorable ring stacked arrangements of mimosines, with interaction energies of mimosine stacked structures significantly higher than analog Phe or Tyr arrangements. This is due to a combined effect between inherent larger interaction energy for large-dipole mimosine rings and a suitable hydrogen bond network with water molecules.

We hope that the parameter sets that we have produced are helpful in future investigations involving this amino acid, with highly promising properties of biomedical relevance. For this reason, we have made the parameters available in Gromacs format at https://osf . io/gfdjh/. Although both the ff99SB and ff03 force fields are known to have biases towards right-handed $\alpha$-helical or $\beta$-sheet conformations, ${ }^{33}$ the parameters that we have produced may serve as a useful starting point for future modelling efforts including more recent modifications. Also, the observation of general trends in two force fields of the Amber family with opposite biases, suggest that our conclusions about the amino acid are robust and likely to be due to the particular chemistry of 
mimosine.

\section{Acknowledgement}

The authors gratefully acknowledge the financing of the MINECO project (PGC2018-099321-BI00) founded by the Spanish Ministry of Economy and Business. A.U. thankfully acknowledges the University of the Basque Country for the scholarship for the completion of a master's degree in the academic year 2018/2019 and the Spanish MECD for a FPU fellowship (FPU 2017/05417). We also acknowledge the Donostia International Physics Center for a summer internship.

\section{Supporting Information Available}

Additional details on the parametrization.

\section{References}

(1) Nguyen, B. C. Q.; Tawata, S. The Chemistry and Biological Activities of Mimosine: A Review. Phytother. Res. 2016, 30, 1230-1242.

(2) Lalande, M. A reversible arrest point in the late G1 phase of the mammalian cell cycle. Exp. Cell Res. 1990, 186, 332-339.

(3) Anitha, R.; Jayavelu, S.; Murugesan, K. Antidermatophytic and bacterial activity of mimosine. Phytother. Res. 2005, 19, 992-993.

(4) Upadhyay, A.; Chompoo, J.; Taira, N.; Fukuta, M.; Gima, S.; Tawata, S. Solid-phase synthesis of mimosine tetrapeptides and their inhibitory activities on neuraminidase and tyrosinase. J. Agric. Food Chem. 2011, 59, 12858-12863. 
(5) Kulp, K. S.; Vulliet, P. R. Mimosine blocks cell cycle progression by chelating iron in asynchronous human breast cancer cells. Toxicol. Appl. Pharmacol. 1996, 139, 356-364.

(6) Gautam, R.; Jachak, S. M.; Kumar, V.; Mohan, C. G. Synthesis, biological evaluation and molecular docking studies of stellatin derivatives as cyclooxygenase (COX-1, COX-2) inhibitors and anti-inflammatory agents. Bioorganic Med. Chem. Lett. 2011, 21, 1612-1616.

(7) Mujika, J.; Dalla Torre, G.; Lachowicz, J.; Lopez, X. In silico design of mimosine containing peptides as new efficient chelators of aluminum. $R S C A d v . \mathbf{2 0 1 9}, 9,7688-7697$.

(8) Nurchi, V. M.; Crisponi, G.; Pivetta, T.; Donatoni, M.; Remelli, M. Potentiometric, spectrophotometric and calorimetric study on iron (III) and copper (II) complexes with 1, 2dimethyl-3-hydroxy-4-pyridinone. J. Inorg. Biochem. 2008, 102, 684-692.

(9) Lloyd, J. B.; Cable, H.; Rice-Evans, C. Evidence that desferrioxamine cannot enter cells by passive diffusion. Biochem. Pharmacol. 1991, 41, 1361-1363.

(10) Lachowicz, J. I.; Dalla Torre, G.; Cappai, R.; Randaccio, E.; Nurchi, V. M.; Bachor, R.; Szewczuk, Z.; Jaremko, L.; Jaremko, M.; Pisano, M. B., et al. Metal self-assembly mimosine peptides with enhanced antimicrobial activity: towards a new generation of multitasking chelating agents. Dalton Trans. 2020, 49, 2862-2879.

(11) Best, R. B. Computational and theoretical advances in studies of intrinsically disordered proteins. Curr. Opin. Struct. Biol. 2017, 42, 147-154.

(12) Hornak, V.; Abel, R.; Okur, A.; Strockbine, B.; Roitberg, A.; Simmerling, C. Comparison of multiple Amber force fields and development of improved protein backbone parameters. Proteins: Struct., Funct., Bioinf. 2006, 65, 712-725.

(13) Duan, Y.; Wu, C.; Chowdhury, S.; Lee, M. C.; Xiong, G.; Zhang, W.; Yang, R.; Cieplak, P.; Luo, R.; Lee, T., et al. A point-charge force field for molecular mechanics simulations of pro- 
teins based on condensed-phase quantum mechanical calculations. J. Comput. Chem. 2003, 24, 1999-2012.

(14) Case, D. et al. AMBER 14. 2014.

(15) Humphrey, W.; Dalke, A.; Schulten, K. VMD: visual molecular dynamics. J. Mol. Graph. 1996, 14, 33-38.

(16) Parrinello, M.; Rahman, A. Polymorphic transitions in single crystals: A new molecular dynamics method. J. Appl. Phys. 1981, 52, 7182-7190.

(17) Essmann, U.; Perera, L.; Berkowitz, M. L.; Darden, T.; Lee, H.; Pedersen, L. G. A smooth particle mesh Ewald method. J. Chem. Phys. 1995, 103, 8577-8593.

(18) Jorgensen, W. L.; Chandrasekhar, J.; Madura, J. D.; Impey, R. W.; Klein, M. L. Comparison of simple potential functions for simulating liquid water. J. Chem. Phys. 1983, 79, 926-935.

(19) Abraham, M. J.; Murtola, T.; Schulz, R.; Páll, S.; Smith, J. C.; Hess, B.; Lindahl, E. GROMACS: High performance molecular simulations through multi-level parallelism from laptops to supercomputers. SoftwareX 2015, 1, 19-25.

(20) Bonomi, M.; Branduardi, D.; Bussi, G.; Camilloni, C.; Provasi, D.; Raiteri, P.; Donadio, D.; Marinelli, F.; Pietrucci, F.; Broglia, R. A., et al. PLUMED: A portable plugin for free-energy calculations with molecular dynamics. Comput. Phys. Commun. 2009, 180, 1961-1972.

(21) Hunter, J. D. Matplotlib: A 2D graphics environment. Comput. Sci. Eng. 2007, 9, 90-95.

(22) Frisch, M. J. et al. Gaussian 16 Rev. A.03. 2016.

(23) Chai, J. D.; Head-Gordon, M. Long-range corrected hybrid density functionals with damped atom-atom dispersion corrections. Phys. Chem. Chem. Phys. 2008, 10, 6615-6620. 
(24) Pritchard, B. P.; Altarawy, D.; Didier, B.; Gibson, T. D.; Windus, T. L. New Basis Set Exchange: An Open, Up-to-Date Resource for the Molecular Sciences Community. J. Chem. Inf. Model. 2019, 59, 4814-4820.

(25) Ditchfield, R.; Hehre, W. J.; Pople, J. A. Self-Consistent Molecular-Orbital Methods. IX. An Extended Gaussian-Type Basis for Molecular-Orbital Studies of Organic Molecules. J. Chem. Phys. 1971, 54, 724-728.

(26) Hehre, W. J.; Ditchfield, R.; Pople, J. A. Self-Consistent Molecular Orbital Methods. XII. Further Extensions of Gaussian-Type Basis Sets for Use in Molecular Orbital Studies of Organic Molecules. J. Chem. Phys. 1972, 56, 2257-2261.

(27) Hariharan, P. C.; Pople, J. A. The influence of polarization functions on molecular orbital hydrogenation energies. Theor. Chim. Acta 1973, 28, 213-222.

(28) Clark, T.; Chandrasekhar, J.; Spitznagel, G. W.; Schleyer, P. V. R. Efficient diffuse functionaugmented basis sets for anion calculations. III. The 3-21+G basis set for first-row elements, Li-F. J. Comput. Chem. 1983, 4, 294-301.

(29) Gordon, M. S.; Binkley, J. S.; Pople, J. A.; Pietro, W. J.; Hehre, W. J. Self-consistent molecular-orbital methods. 22. Small split-valence basis sets for second-row elements. J. Am. Chem. Soc. 1982, 104, 2797-2803.

(30) Francl, M. M.; Pietro, W. J.; Hehre, W. J.; Binkley, J. S.; Gordon, M. S.; DeFrees, D. J.; Pople, J. A. Self-consistent molecular orbital methods. XXIII. A polarization-type basis set for second-row elements. J. Chem. Phys. 1982, 77, 3654-3665.

(31) Spitznagel, G. W.; Clark, T.; Schleyer, P. v. R.; Hehre, W. J. An evaluation of the performance of diffuse function-augmented basis sets for second row elements, Na-Cl. J. Comput. Chem. 1987, 8, 1109-1116. 
(32) Tomasi, J.; Mennucci, B.; Cammi, R. Quantum Mechanical Continuum Solvation Models. Chem. Rev. 2005, 105, 2999-3094.

(33) Best, R. B.; Hummer, G. Optimized molecular dynamics force fields applied to the helix- coil transition of polypeptides. J. Phys. Chem. B 2009, 113, 9004-9015. 